\title{
SELF-SIMILAR SOLUTIONS OF KINETIC-TYPE EQUATIONS: THE BOUNDARY CASE
}

\author{
KAMIL BOGUS, DARIUSZ BURACZEWSKI AND ALEXANDER MARYNYCH
}

\begin{abstract}
For a time dependent family of probability measures $\left(\rho_{t}\right)_{t \geqslant 0}$ we consider a kinetic-type evolution equation $\partial \phi_{t} / \partial t+\phi_{t}=\widehat{Q} \phi_{t}$ where $\widehat{Q}$ is a smoothing transform and $\phi_{t}$ is the Fourier-Stieltjes transform of $\rho_{t}$. Assuming that the initial measure $\rho_{0}$ belongs to the domain of attraction of a stable law, we describe asymptotic properties of $\rho_{t}$, as $t \rightarrow \infty$. We consider the boundary regime when the standard normalization leads to a degenerate limit and find an appropriate scaling ensuring a non-degenerate self-similar limit. Our approach is based on a probabilistic representation of probability measures $\left(\rho_{t}\right)_{t \geqslant 0}$ that refines the corresponding construction proposed in Bassetti and Ladelli [Ann. Appl. Probab. 22(5): 1928-1961, 2012].
\end{abstract}

\section{INTRODUCTION}

In the paper we consider a kinetic-type evolution equation for a time dependent family of probability measures $\left(\rho_{t}\right)_{t \geqslant 0}$. Let

$$
\phi(t, \xi)=\int_{\mathbb{R}} e^{i \xi v} \rho_{t}(\mathrm{~d} v), \quad t \geqslant 0, \quad \xi \in \mathbb{R},
$$

be the Fourier-Stieltjes transform (the characteristic function) of $\rho_{t}$. We are interested in the solution of the following Cauchy problem

$$
\frac{\partial}{\partial t} \phi(t, \xi)+\phi(t, \xi)=\widehat{Q}(\phi(t, \cdot), \ldots, \phi(t, \cdot))(\xi), \quad t>0, \quad \phi(0, \xi)=\phi_{0}(\xi), \quad \xi \in \mathbb{R},
$$

where $\widehat{Q}$ is a smoothing transform. The smoothing transform $\widehat{Q}$ is defined by the equality

$$
\widehat{Q}\left(\phi_{1}, \ldots, \phi_{N}\right)(\xi):=\mathbb{E}\left(\phi_{1}\left(A_{1} \xi\right) \cdot \ldots \cdot \phi_{N}\left(A_{N} \xi\right)\right), \quad \xi \in \mathbb{R},
$$

where $\phi_{1}, \ldots, \phi_{N}$ are characteristic functions, $N$ is a fixed positive integer, and a random vector $\mathbb{A}=\left(A_{1}, \ldots, A_{N}\right)$ consists of positive real-valued random variables defined on a common probability space $(\Omega, \mathcal{F}, \mathbb{P})$. The initial condition $\phi_{0}$ is the characteristic function of some random variable $X_{0}$ defined on $(\Omega, \mathcal{F}, \mathbb{P})$.

The equation of the form (1.1) with $N=2$ and $\mathbb{A}=(\sin \theta, \cos \theta)$, where $\theta$ is a random angle uniformly distributed on $[0,2 \pi)$, was introduced and investigated by Kac [25] as a model of behavior of a particle in a homogeneous gas. In subsequent works the Kac model

2010 Mathematics Subject Classification. 60F05, 82C40.

Key words and phrases. Biggins martingale, derivative martingale, Kac model, kinetic equation, random trees, smoothing transform.

K. Bogus and D. Buraczewski were partially supported by the National Science Center, Poland (Sonata Bis, grant number DEC-2014/14/E/ST1/00588). A. Marynych was partially supported by the Return Fellowship of the Alexander von Humboldt Foundation. 
was generalized in various directions including one dimensional dissipative Maxwell models for colliding molecules [30], models describing economical dynamics [28] and the inelastic Boltzmann equation [14, 15]. We refer to [4, 5, 7] for other examples and a comprehensive bibliography.

In this paper we study asymptotic behavior of the solution $\phi$ to equation (1.1) from probabilistic point of view and prove related limit theorems. This problem was recently addressed in 4 where it was shown that under mild assumptions, which we discuss later, there exists a parameter $\mu$, depending on the initial condition $\phi_{0}$ and the law of $\mathbb{A}$, such that the rescaled solution to (1.1), namely

$$
w(t, \xi)=\phi\left(t, e^{-\mu t} \xi\right), \quad t \geqslant 0, \quad \xi \in \mathbb{R},
$$

converges to a nondegenerate limit as $t \rightarrow \infty$ and the limit is a fixed point of a smoothing transform pertained to $\widehat{Q}$. The main goal of our paper is to present a class of solutions to (1.1) which, after rescaling as in (1.2), converge to a degenerate limit, yet it is possible to find a different normalization ensuring a nondegenerate limit possessing some self-similarity properties. To achieve our aims we propose a refinement of the probabilistic construction of the solution $\phi$ presented in [5] and express $\phi$ via a continuous-time branching random walk.

Firstly, we state assumptions concerning the initial condition $\phi_{0}$. We suppose, similarly as in [4] and [7], that the distribution function $F_{0}$ of $X_{0}$ satisfies one of the following hypotheses $\left(H_{\gamma}\right)$ for some $\gamma \in(0,2]$ :

$\left(H_{1}\right)$ either

(a) $\int_{\mathbb{R}}|v| \mathrm{d} F_{0}(v)<+\infty$ and then we set $m_{0}:=\int_{\mathbb{R}} v \mathrm{~d} F_{0}(v)$

or

(b) $F_{0}$ satisfies the conditions

$$
\lim _{x \rightarrow+\infty} x\left(1-F_{0}(x)\right)=\lim _{x \rightarrow-\infty}|x| F_{0}(x)=c_{0}^{+} \in(0, \infty),
$$

and

$$
\lim _{R \rightarrow+\infty} \int_{-R}^{R} v \mathrm{~d} F_{0}(v)=: m_{0} \in(-\infty, \infty) .
$$

$\left(H_{2}\right) 0<\sigma_{0}^{2}:=\int_{\mathbb{R}}|v|^{2} \mathrm{~d} F_{0}(v)<+\infty$ and $\int_{\mathbb{R}} v \mathrm{~d} F_{0}(v)=0$.

$\left(H_{\gamma}\right)$ If $\gamma \in(0,1) \cup(1,2), F_{0}$ satisfies the conditions

$$
\lim _{x \rightarrow+\infty} x^{\gamma}\left(1-F_{0}(x)\right)=c_{0}^{+}<+\infty, \quad \lim _{x \rightarrow-\infty}|x|^{\gamma} F_{0}(x)=c_{0}^{-}<+\infty
$$

with $c_{0}^{+}+c_{0}^{-}>0$ and, in addition, $\int_{\mathbb{R}} v \mathrm{~d} F_{0}(v)=0$ if $\gamma \in(1,2)$.

Further, we define the function $\hat{g}_{\gamma}: \mathbb{R} \mapsto \mathbb{C}$ by

$$
\hat{g}_{\gamma}(\xi):= \begin{cases}e^{i m_{0} \xi}, & \text { if } \gamma=1 \text { and }(\mathrm{a}) \text { of }\left(H_{1}\right) \text { holds, } \\ e^{i m_{0} \xi-\pi c_{0}^{+}|\xi|}, & \text { if } \gamma=1 \text { and }(\mathrm{b}) \text { of }\left(H_{1}\right) \text { holds, } \\ e^{-\sigma_{0}^{2}|\xi|^{2} / 2}, & \text { if } \gamma=2 \text { and }\left(H_{2}\right) \text { holds, } \\ e^{-k_{0}|\xi|^{\gamma}\left(1-i \eta_{0} \tan (\pi \gamma / 2) \operatorname{sign} \xi\right)}, & \text { if } \gamma \in(0,1) \cup(1,2) \text { and }\left(H_{\gamma}\right) \text { holds, }\end{cases}
$$

where

$$
k_{0}=\left(c_{0}^{+}+c_{0}^{-}\right) \frac{\pi}{2 \Gamma(\gamma) \sin (\pi \gamma / 2)}, \quad \eta_{0}=\frac{c_{0}^{+}-c_{0}^{-}}{c_{0}^{+}+c_{0}^{-}}
$$


Observe that the condition $\left(H_{\gamma}\right)$ is equivalent to the fact that the law of $X_{0}$ (the law of $X_{0}-m_{0}$ in case $\left.H_{1}(b)\right)$ is centered and (except case $H_{1}(a)$ ) belongs to the domain of normal attraction of a $\gamma$-stable law with the characteristic function $\hat{g}_{\gamma}$, see a concluding remark on p. 581 in [19, Chapter XVIII.5].

Now we formulate our hypotheses on the smoothing transform $\widehat{Q}$. Our first assumption is that the weights $\left(A_{i}\right)_{i=1, \ldots, N}$ are a.s. positive. Next we define the function $\Phi:[0, \infty) \mapsto$ $\mathbb{R} \cup\{+\infty\}$ via

$$
\Phi(s)=\mathbb{E}\left[\sum_{i=1}^{N} A_{i}^{s}\right]-1, \quad s \geqslant 0,
$$

and assume that $s_{\infty}>0$ where $s_{\infty}:=\sup \{s \geqslant 0: \Phi(s)<\infty\}$. Note that the function $\Phi$ is smooth and convex on $\left(0, s_{\infty}\right)$. The function

$$
\mu(s)=\frac{\Phi(s)}{s}, \quad s>0,
$$

is called spectral function, see [14]. Observe that $\mu(s)$ is equal to the tangent of the angle between the vector joining $(0,0)$ and $(s, \Phi(s))$ and the positive horizontal half-axis. Since $\Phi$ is strictly convex and smooth there exists exactly one point $\gamma^{*}$ minimizing the spectral function, and then the corresponding line is just tangent to the function $\Phi$ at point $\left(\gamma^{*}, \Phi\left(\gamma^{*}\right)\right)$, see Fig. 1. Moreover, $\mu\left(\gamma^{*}\right)=\Phi^{\prime}\left(\gamma^{*}\right)$.

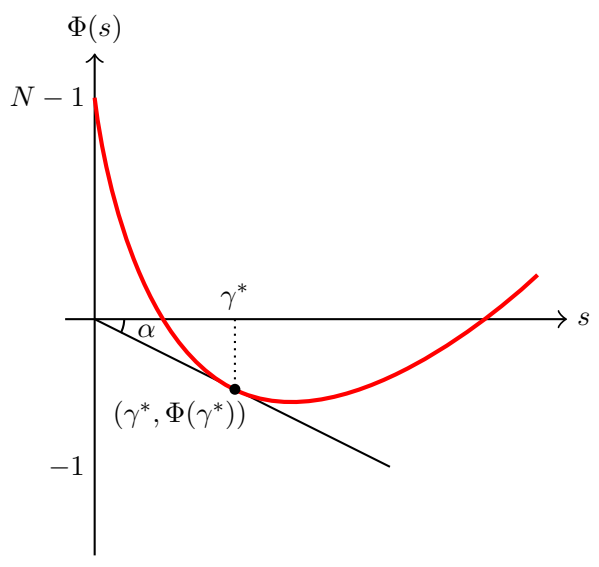

Figure 1. Plot of the function $s \mapsto \Phi(s)$ (solid red) with $\tan \alpha=\mu\left(\gamma^{*}\right)=$ $\Phi^{\prime}\left(\gamma^{*}\right)=\Phi\left(\gamma^{*}\right) / \gamma^{*}$.

In the series of papers [4, 5] Bassetti, Ladelli and Matthes found a probabilistic interpretation of the solution $\phi$ via labelled random trees. Assuming that $\left(H_{\gamma}\right)$ holds for some $\gamma \in(0,2]$ and there exists $\delta>\gamma$ such that $\mu(\delta)<\mu(\gamma)<\infty$, it is shown in [4, Theorem $2.2]$ that $\phi\left(t, e^{-\mu(\gamma) t} \xi\right)$ converges to a nondegenerate limit being the characteristic function of the law of the limit of some positive martingale related to a family of random labelled trees. Clearly, if $\gamma=\arg \min \mu(s)$ no such $\delta$ exists and, moreover, it can be checked that the corresponding martingale converges to 0 . As manifested in the title of the paper and motivated by Biggins and Kyprianou [13, who considered the smoothing transform in the case $\gamma^{*}=1$ and $\mu\left(\gamma^{*}\right)=0$, we call this situation the boundary case. 
The main result of our paper is given by Theorem 1.4 below, and provides the correct normalization in the boundary case leading to a non-degenerate limit. As we will see, the right normalization involves a subexponential term and the limit is a fixed point of some smoothing transform.

Theorem 1.4. Assume that for some $\gamma \in(0,2]$ the hypothesis $\left(H_{\gamma}\right)$ is satisfied and

$$
\gamma=\underset{s \in\left(0, s_{\infty}\right)}{\arg \min } \mu(s)=\gamma^{*} \in\left(0, s_{\infty}\right) .
$$

Then there exists a probability measure $\rho_{\infty}$ such that the function $\phi$, the unique solution to (1.1), satisfies

$$
\lim _{t \rightarrow \infty} \phi\left(t, t^{\frac{1}{2 \gamma}} e^{-\mu(\gamma) t} \xi\right)=w_{\infty}(\xi), \quad \xi \in \mathbb{R}
$$

where $w_{\infty}$ is the Fourier-Stieltjes transform of $\rho_{\infty}$. Moreover, $w_{\infty}$ has the following representation $w_{\infty}(\xi)=\mathbb{E} \widehat{g}_{\gamma}\left(\xi c_{\gamma} D_{\infty}^{1 / \gamma}\right)$, where $c_{\gamma}:=\left(\frac{2}{\pi \gamma^{2} \Phi^{\prime \prime}(\gamma)}\right)^{\frac{1}{2 \gamma}}$ and $D_{\infty}$ is a.s. positive random variable defined in Proposition 3.2 below and which satisfies the following stochastic fixed-point equation

$$
D_{\infty} \stackrel{d}{=} \mathcal{U}^{\Phi(\gamma)} \sum_{k=1}^{N} A_{k}^{\gamma} D_{\infty}^{(k)},
$$

where $\left(D_{\infty}^{(k)}\right)_{k=1}^{N}$ are independent copies of $D_{\infty} ; \mathcal{U}$ has a uniform distribution on $(0,1)$ and $\left(D_{\infty}^{(k)}\right)_{k=1}^{N}, \mathcal{U}$ and $\left(A_{1}, \ldots, A_{N}\right)$ are independent.

The rest of the paper is organized as follows. In Section 2 we describe a probabilistic representation of the solution $\phi$, which essentially reminds the construction in [4] but is more transparent and convenient for the analysis. Moreover, we reveal some further probabilistic structure behind this construction by pointing out a connection to Yule processes and branching random walks in continuous time. We strongly believe that the representation proposed in Section 2 is the most accurate probabilistic interpretation of the solution to equation (1.1). In Section 3 we prove a convergence result for the Biggins martingale in continuous time branching random walk and explain the construction of the limiting measure $\rho_{\infty}$. The proof of Theorem 1.4 is given in Section 4 .

\section{Probabilistic Representation of the solution}

The solution to the equation (1.1) can be derived analytically in terms of the Wild series [33, see also Kielek [26]. However, based on McKean's [29] ideas, Bassetti, Ladelli and Matthes [4, 5] expressed the solution in a convenient probabilistic way. Ealier results on probabilistic representation can be found in [16, 20, 21].

The probabilistic construction of the solution $\phi$ using labelled $N$-ary random trees is given on pp. 1938-1939 of [4] see Proposition 3.2 therein, where the authors use among other a stochastic process called $\left(\nu_{t}\right)_{t \geqslant 0}$. However, it is defined as an arbitrary stochastic process with specified marginal distributions, see the top of p. 1939 in [4]. Even though such specification is sufficient for the asymptotic analysis of $\phi$, it leaves an open and interesting question of finding a correct interpretation and pathwise construction of $\left(\nu_{t}\right)_{t \geqslant 0}$. The main purpose of this subsection is to propose a natural representation of $\left(\nu_{t}\right)_{t \geqslant 0}$ and to provide 
an alternative form of Proposition 3.2 of [4] revealing the complete probabilistic structure of the solution $\phi$. As we will see, $\phi(t, \cdot)$ is nothing else but the characteristic function of a smoothing transform associated with a certain continuous-time branching random walk and applied to the distribution of $X_{0}$, see Proposition 2.5 below.

2.1. Representation of the solutions and connection with branching random walks in continuous time. Let us recall that a Yule process $\left(\mathcal{Y}_{t}\right)_{t \geqslant 0}$ is a pure birth process which starts with one particle. After exponential time with parameter 1 the original particle dies out and produces $N$ new particles. Every particle behaves as the original one, and the particles reproduce independently. The quantity $\mathcal{Y}_{t}$ is the number of particles at time $t \geqslant 0$. Denote by $F(s, t)$ the probability generating function of $\mathcal{Y}_{t}$, that is

$$
F(s, t)=\mathbb{E} s^{\mathcal{Y}_{t}}, \quad t \geqslant 0, \quad|s| \leqslant 1 .
$$

Using equations (5) and (6) on p. 106 in [3], see also example on p. 109 in the same reference, we obtain

$$
\frac{\partial F(s, t)}{\partial t}=F^{N}(s, t)-F(s, t), \quad t>0, \quad F(s, 0)=s .
$$

By solving this differential equation, we get the explicit solution

$$
F(s, t)=s\left(\frac{e^{-(N-1) t}}{1-s^{N-1}\left(1-e^{-(N-1) t}\right)}\right)^{\frac{1}{N-1}}, \quad t \geqslant 0, \quad|s| \leqslant 1 .
$$

The full genealogical tree $\mathcal{T}_{\infty}$ of the Yule process $\left(\mathcal{Y}_{t}\right)_{t \geqslant 0}$ is an infinite $N$-ary random tree. For every fixed $T \geqslant 0$ the genealogical tree of $\left(\mathcal{Y}_{t}\right)_{t \in[0, T]}$ is a finite $N$-ary random tree with leaves representing the particles alive at time $T$ and internal nodes being the particles which have died out during $[0, T]$. Denote the number of latter particles by $\nu_{T}$. We have the following identity

$$
\mathcal{Y}_{t}=(N-1) \nu_{t}+1, \quad t \geqslant 0 .
$$

From this representation and formula (2.1) we get

$$
\begin{aligned}
& \mathbb{E} s^{\nu t}=e^{-t}\left(1-s\left(1-e^{-(N-1) t}\right)\right)^{-\frac{1}{N-1}} \\
& =\sum_{k \geqslant 0} \frac{\Gamma\left(\frac{1}{N-1}+k\right)}{k ! \Gamma\left(\frac{1}{N-1}\right)} e^{-t}\left(1-e^{-(N-1) t}\right)^{k} s^{k}, \quad t \geqslant 0, \quad|s| \leqslant 1,
\end{aligned}
$$

in full agreement with formula (3.4) in [4]. That is to say, the process $\left(\nu_{t}\right)_{t \geqslant 0}$ introduced in [4], should be interpreted as the number of splits during the time interval $[0, t]$ in the Yule process $\left(\mathcal{Y}_{t}\right)_{t \geqslant 0}$. This interpretation of the distribution of $\nu_{t}$ is the starting point of our probabilistic construction of the solution $\phi$.

By adding to the definition of a Yule process the control over positions of particles, we obtain a continuous-time branching random walk introduced in [32]. More precisely, let $\zeta=\sum_{k=1}^{N} \delta_{Z_{k}}$ be an arbitrary point process on $\mathbb{R}$, where $\delta_{x}$ denotes the Dirac point measure at $x \in \mathbb{R}$. In the continuous-time branching random walk the initial single particle is located at 0 . After an exponential time with parameter 1 it dies out and gives birth to $N$ new particles which are placed at positions $\left(Z_{1}, \ldots, Z_{N}\right)$. These particles reproduce independently in the same fashion as their mother. In particular, if at any time a particle 
$v$ located at some $x \in \mathbb{R}$ splits, its children are placed at $x+Z_{1}(v), \ldots, x+Z_{N}(v)$, where $\zeta^{(v)}:=\sum_{k=1}^{N} \delta_{Z_{k}(v)}$ is an independent copy of $\zeta$. In what follows we only consider branching random walks with deterministic number of children of every particle. Clearly, the number of particles in such a continuous-time branching random walk at time $t \geqslant 0$ is just $\mathcal{Y}_{t}$. Denote the locations of particles present at time $t$ by $z_{1, t}, z_{2, t}, \ldots, z_{\mathcal{Y}_{t}, t}$. The continuoustime branching random walk is formally defined as the measure-valued stochastic process

$$
\mathcal{Z}_{t}:=\sum_{k=1}^{\mathcal{Y}_{t}} \delta_{z_{k, t}}, \quad t \geqslant 0 .
$$

It will be important that the process $\left(\mathcal{Z}_{t}\right)_{t \geqslant 0}$ satisfies the following branching relation:

$$
\mathcal{Z}_{t+s}(\cdot) \stackrel{d}{=} \sum_{k=1}^{\mathcal{Y}_{t}} \mathcal{Z}_{s}^{(k)}\left(\cdot-z_{k, t}\right), \quad t, s \geqslant 0
$$

where $\left(\mathcal{Z}_{t}^{(k)}\right)_{t \geqslant 0}$ for $k \in \mathbb{N}$ are independent copies of $\left(\mathcal{Z}_{t}\right)_{t \geqslant 0}$.

Finally, given a continuous-time branching random walk $\left(\mathcal{Z}_{t}\right)_{t \geqslant 0}$, the associated family of smoothing transforms $\left(\mathcal{L}_{t}^{(\gamma)}\right)_{t \geqslant 0}$ on the space of probability distributions on $\mathbb{R}$ is defined by

$$
\mathcal{L}_{t}^{(\gamma)}(\operatorname{distr}(U))=\operatorname{distr}\left(\sum_{k=1}^{\mathcal{Y}_{t}} e^{\gamma z_{k, t}} U_{k}\right),
$$

where $\left(U_{k}\right)_{k \geqslant 1}$ are independent copies of a random variable $U$ and $\gamma \in \mathbb{C}$ is a parameter. By slightly abusing notation we write $\mathcal{L}_{t}^{(\gamma)}(U)$ instead of $\mathcal{L}_{t}^{(\gamma)}(\operatorname{distr}(U))$. We also suppress the index $\gamma$ if it is equal to 1 by writing $\mathcal{L}_{t}$ instead of $\mathcal{L}_{t}^{(1)}$.

We are ready to state the main result of this subsection, namely the probabilistic representation of the solution $\phi$ to kinetic-type equation (1.1). Assume that on the probability space $(\Omega, \mathcal{F}, \mathbb{P})$ the following two objects are defined:

- the continuous time branching random walk $\left(\mathcal{Z}_{t}\right)_{t \geqslant 0}$ with the displacement process $\zeta:=\sum_{k=1}^{N} \delta_{\log A_{k}}:$

$$
\mathcal{Z}_{t}:=\sum_{k=1}^{\mathcal{Y}_{t}} \delta_{z_{k, t}}, \quad t \geqslant 0 .
$$

- the sequence $\left(X_{k}\right)_{k \geqslant 1}$ of independent random variables with common distribution function $F_{0}$, which is also independent of $\left(\mathcal{Z}_{t}\right)_{t \geqslant 0}$.

Proposition 2.5. Equation (1.1) has a unique solution $\phi(t, \cdot)$ which is given by

$$
\phi(t, \xi)=\mathbb{E} \exp \left(i \xi\left(\sum_{k=1}^{\mathcal{Y}_{t}} e^{z_{k, t}} X_{k}\right)\right), \quad t \geqslant 0, \quad \xi \in \mathbb{R},
$$

that is $\phi(t, \cdot)$ is the characteristic function of the random variable $\mathcal{L}_{t}\left(X_{0}\right)$, where $\mathcal{L}_{t}$ is the smoothing transform associated with the continuous-time branching random walk $\left(\mathcal{Z}_{t}\right)_{t \geqslant 0}$.

Proof. Uniqueness of the solution follows from a standard use of the Picard-Lindelöf theorem, see e.g. proof of Proposition 2.2 in [6]. Thus it is enough to show that the right-hand 
side of (2.6) satisfies (1.1). To this end, denote the the right-hand side of (2.6) by $\psi(t, \xi)$ and write

$$
\psi(t, \xi)=\mathbb{E}\left[\prod_{k=1}^{\mathcal{Y}_{t}} \phi_{0}\left(\xi e^{z_{k, t}}\right)\right]=\mathbb{E} \exp \left(\int_{\mathbb{R}} \log \phi_{0}\left(\xi e^{y}\right) \mathcal{Z}_{t}(\mathrm{~d} y)\right), \quad t \geqslant 0, \quad \xi \in \mathbb{R} .
$$

Firstly, let us show that $t \mapsto \psi(t, \xi)$ is continuous for every fixed $\xi$. For $t, s \geqslant 0$ we can write

$$
|\psi(t, \xi)-\psi(s, \xi)| \leqslant 2 \mathbb{P}\{\text { there are splits during }[t \wedge s, t \vee s]\}=2 \mathbb{E}\left(1-e^{-\mathcal{Y}_{t \wedge s}|t-s|}\right) \rightarrow 0
$$

as $s \rightarrow t$, by the dominated convergence theorem and the observation $\mathcal{Y}_{t}<\infty$ a.s.

Further, for $t \geqslant 0$, let $\mathcal{F}_{t} \subset \mathcal{F}$ be the $\sigma$-algebra generated by $\left(\mathcal{Z}_{s}\right)_{s \in[0, t]}$. For $h \geqslant 0$, using formula (2.4), we obtain

$$
\begin{aligned}
\psi(t+h, \xi) & =\mathbb{E}\left(\mathbb{E}\left(\exp \left(\int_{\mathbb{R}} \log \phi_{0}\left(\xi e^{y}\right) \mathcal{Z}_{t+h}(\mathrm{~d} y)\right) \mid \mathcal{F}_{h}\right)\right) \\
& =\mathbb{E}\left(\mathbb{E}\left(\prod_{k=1}^{\mathcal{Y}_{h}} \exp \left(\int_{\mathbb{R}} \log \phi_{0}\left(\xi e^{y} e^{z_{k, h}}\right) \mathcal{Z}_{t}^{(k)}(\mathrm{d} y)\right) \mid \mathcal{F}_{h}\right)\right) \\
& =\mathbb{E}\left(\prod_{k=1}^{\mathcal{Y}_{h}} \mathbb{E}\left(\exp \left(\int_{\mathbb{R}} \log \phi_{0}\left(\xi e^{y} e^{z_{k, h}}\right) \mathcal{Z}_{t}^{(k)}(\mathrm{d} y)\right) \mid \mathcal{F}_{h}\right)\right)=\mathbb{E}\left(\prod_{k=1}^{\mathcal{Y}_{h}} \psi\left(t, \xi e^{z_{k, h}}\right)\right) .
\end{aligned}
$$

The probability of having two or more splits in the branching random walk $\left(\mathcal{Z}_{t}\right)_{t \geqslant 0}$ during $[0, h]$ is $o(h)$ as $h \rightarrow+0$, whence

$$
\begin{aligned}
\psi(t+h, \xi) & =\mathbb{E}\left(\prod_{k=1}^{\mathcal{Y}_{h}} \psi\left(t, \xi e^{z_{k, h}}\right)\right) \\
& =\psi(t, \xi) \mathbb{P}\{\text { there are no splits during }[0, h]\} \\
& +\mathbb{E}\left(\prod_{j=1}^{N} \psi\left(t, \xi A_{j}\right)\right) \mathbb{P}\{\text { there is exactly one split during }[0, h]\}+o(h) \\
& =\psi(t, \xi) e^{-h}+\mathbb{E}\left(\prod_{j=1}^{N} \psi\left(t, \xi A_{j}\right)\right) h+o(h) .
\end{aligned}
$$


Likewise, we can write for $h \geqslant 0$ and $t \geqslant h$ :

$$
\begin{aligned}
\psi(t, \xi) & =\mathbb{E}\left(\prod_{k=1}^{\mathcal{Y}_{h}} \psi\left(t-h, \xi e^{z_{k, h}}\right)\right) \\
& =\psi(t-h, \xi) \mathbb{P}\{\text { there are no splits during }[0, h]\} \\
& +\mathbb{E}\left(\prod_{j=1}^{N} \psi\left(t-h, \xi A_{j}\right)\right) \mathbb{P}\{\text { there is exactly one split during }[0, h]\}+o(h) \\
& =\psi(t-h, \xi) e^{-h}+\mathbb{E}\left(\prod_{j=1}^{N} \psi\left(t-h, \xi A_{j}\right)\right) h+o(h) .
\end{aligned}
$$

Rearranging the terms and sending $h \rightarrow+0$ shows that

$$
\frac{\partial \psi(t, \xi)}{\partial t}+\psi(t, \xi)=\mathbb{E}\left(\prod_{j=1}^{N} \psi\left(t, \xi A_{j}\right)\right)=\widehat{Q}(\psi(t, \cdot), \ldots, \psi(t, \cdot)), \quad t>0
$$

by the dominated convergence theorem and continuity of $t \mapsto \psi(t, \xi)$ (this is required for the left derivative). Therefore, $\psi$ is a solution to (1.1). Since the solution is unique and $\psi(0, \xi)=\phi_{0}(\xi)=\phi(0, \xi)$, we infer $\psi(t, \xi) \equiv \phi(t, \xi)$. The proof is complete.

Remark 2.7. Let us now compare our Proposition 2.5] with Proposition 3.2 in [4] in more details. Proposition 3.2 in [4] states that the unique solution $\phi$ to (1.1) is

$$
\phi(t, \xi)=\int_{\mathbb{R}} e^{i \xi v} \rho_{t}(\mathrm{~d} v)=\int_{\mathbb{R}} e^{i \xi v} \mathbb{P}\left\{W_{\nu_{t}} \in \mathrm{d} v\right\},
$$

where $\nu_{t}$ is an integer-valued random variable with the generating function (2.3) and which is independent of $\left(W_{n}\right)_{n \geqslant 0}$. For $n=0,1,2, \ldots, W_{n}$ is defined by a sum

$$
W_{n}:=\sum_{i=1}^{(N-1) n+1} \omega\left(v_{i, n}\right) X_{v_{i, n}}
$$

where $v_{i, n}, i=1, \ldots,(N-1) n+1$ are the leaves of a random labelled $N$-ary recursive tree $\mathcal{T}_{n}$ after $n$ steps, $\omega(v)$ is the weight of a leaf $v \in \mathcal{T}_{n}$ (the product of all labels on the unique path from the root to $v$ ), and $\left(X_{v}\right)$ is a family of independent random variables with common distribution function $F_{0}$ which is also independent of the random labelled tree $\mathcal{T}_{n}$. Our construction described above unifies and reinterprets all the aforementioned ingredients: the sequence of random $N$-ary trees $\left(\mathcal{T}_{n}\right)$, the labels of their nodes and the subordination time $\nu_{t}$ via a single object, the continuous-time branching random walk $\left(\mathcal{Z}_{t}\right)_{t \geqslant 0}$. We summarize the above observations in Table 1 .

The connection between random $N$-ary trees, Yule processes and branching random walks, is by no means new and have already been observed in probabilistic literature, see for example [17] for the case of binary search trees. Recently this connection has been extensively exploited in the analysis of profiles of random trees in [24].

Remark 2.8. As has been pointed out by the referee our Proposition 2.5 remains valid also with random $N$ such that $\mathbb{E} N \in(1, \infty)$. The latter condition guarantees that $\left(\mathcal{Z}_{t}\right)_{t \geqslant 0}$ 
TABle 1. Comparison of two probabilistic constructions of $\phi$

\begin{tabular}{ll}
\hline The construction in [4, $[5]$ & A counterpart in our construction \\
\hline the sequence of random $N$-ary recursive & the skeleton of the Yule process $\left(\mathcal{Y}_{t}\right)_{t \geqslant 0}$ \\
trees $\left(\mathcal{T}_{n}\right)_{n \geqslant 0}$ & pertained to $\left(\mathcal{Z}_{t}\right)_{t \geqslant 0}$ and observed at split- \\
& ting times \\
\hline labels of the nodes in the trees $\left(\mathcal{T}_{n}\right)_{n \geqslant 0}$ & relative displacements of the particles in \\
& $\left(\mathcal{Z}_{t}\right)_{t \geqslant 0}$ \\
\hline random variables $\nu_{t}, t \geqslant 0$ & random process $\left(\nu_{t}\right)_{t \geqslant 0}$, the number of \\
& splits in $\left(\mathcal{Z}_{s}\right)_{s \geqslant 0}\left(\right.$ or $\left.\left(\mathcal{Y}_{t}\right)_{t \geqslant 0}\right)$ on $[0, t]$ \\
\hline
\end{tabular}

does not explode and has a positive survival probability. On the other hand, probabilistic construction used in [4, 5] does not seem to have a direct analogue for random $N$ due to a lack of explicit distribution for $\nu_{t}$ for a fixed $t>0$.

Last but not least, we would like to emphasize that the main advantage of Proposition 2.5 is its generality. It allows one to translate limit theorems for the smoothing transform $\mathcal{L}_{t}^{(\gamma)}\left(X_{0}\right)$, as $t \rightarrow \infty$, to the corresponding asymptotics for the solution of (1.1), as $t \rightarrow \infty$. In particular, Proposition 2.5 is useful not only in the case considered in our paper - the boundary case - but also in other situations. As we will see in the next sections, limit theorems for $\mathcal{L}_{t}^{(\gamma)}\left(X_{0}\right)$ are intimately connected with convergence in probability of a socalled Biggins martingale for the continuous-time branching random walk $\left(\mathcal{Z}_{t}\right)_{t \geqslant 0}$.

\section{Convergence of the continuous-time Biggins martingale in the boundary} CASE.

For every $\gamma \in\left[0, s_{\infty}\right)$, put

$$
\mathcal{M}_{t}(\gamma):=e^{-\Phi(\gamma) t} \sum_{k=1}^{\mathcal{Y}_{t}} e^{\gamma z_{k, t}}, \quad t \geqslant 0,
$$

and note that by formula (5.1) in [1] we have

$$
\mathbb{E} \mathcal{M}_{t}(\gamma)=1
$$

The stochastic process $\left(\mathcal{M}_{t}(\gamma)\right)_{t \geqslant 0}$ is a martingale and is called continuous-time Biggins martingale.

If $\gamma=\gamma^{*}=\arg \min _{s \in\left[0, s_{\infty}\right)} \mu(s)$ and $\gamma^{*}<s_{\infty}$, then the Biggins martingale $\left(\mathcal{M}_{t}\left(\gamma^{*}\right)\right)_{t \geqslant 0}$ converges to zero a.s. For the discrete-time Biggins martingale this fact is well-known, see, for example, Lemma 5 in [10], and for the continuous-time Biggins martingale it follows from Theorem 1.1 of the recent paper [9] as well as from Proposition 3.2(i) below.

Proposition 3.2. Assume that $\gamma^{*} \in\left(0, s_{\infty}\right)$. The following limit relations hold true.

(i) Ast $\rightarrow \infty$ we have

$$
\sqrt{t} \mathcal{M}_{t}\left(\gamma^{*}\right)=\sqrt{t} \sum_{k=1}^{\mathcal{Y}_{t}} e^{\gamma^{*} z_{k, t}^{\circ}} \stackrel{\mathbb{P}}{\rightarrow} \sqrt{\frac{2}{\pi\left(\gamma^{*}\right)^{2} \Phi^{\prime \prime}\left(\gamma^{*}\right)}} D_{\infty}
$$


where $z_{k, t}^{\circ}=z_{k, t}-t \mu\left(\gamma^{*}\right), D_{\infty}$ is the a.s. limit of the derivative martingale

$$
\mathcal{D}_{t}\left(\gamma^{*}\right):=\sum_{k=1}^{\mathcal{Y}_{t}} e^{\gamma^{*} z_{k, t}^{\circ}} z_{k, t}^{\circ}, \quad t \geqslant 0 .
$$

The random variable $D_{\infty}$ is a.s. positive and satisfies (1.5).

(ii) Moreover,

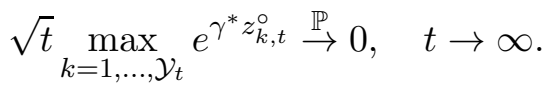

The derivation of Proposition 3.2 utilizes ideas borrowed from [18], where part (i) has been stated without a proof in Remark 2.11(iii). Firstly, we obtain two auxiliary lemmas which show that the Biggins martingale $\left(\mathcal{M}_{t}\left(\gamma^{*}\right)\right)_{t \geqslant 0}$ is in the boundary case. In particular, this implies that every $\theta$-skeleton, that is the discrete-time Biggins martingale $\left(\mathcal{M}_{n \theta}\left(\gamma^{*}\right)\right)_{n \geqslant 0}, \theta>0$, is also in the boundary case. Thereafter, we apply the corresponding theorem by Aïdékon and Shi [2], who found the appropriate normalization for the discretetime Biggins martingales in the boundary case, to our $\theta$-skeletons and then pass to the continuous parameter with the aid of the Croft-Kingman lemma.

Lemma 3.6. Assume that $\gamma^{*} \in\left(0, s_{\infty}\right)$. For every $t \geqslant 0$ we have

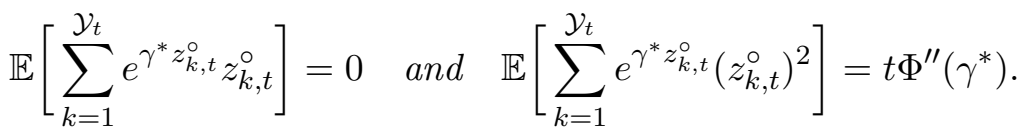

Proof. Fix $\varepsilon \in\left(0, \gamma^{*}\right)$ such that $\gamma^{*}+\varepsilon<s_{\infty}$. Let us show that for every fixed $t \geqslant 0$ the following holds:

$$
\mathbb{E}\left[\sum_{k=1}^{\mathcal{Y}_{t}} e^{\gamma^{*} z_{k, t}} z_{k, t}\right]=\left.\frac{\partial}{\partial \gamma}\left[\mathbb{E} \int_{\mathbb{R}} e^{\gamma y} \mathcal{Z}_{t}(\mathrm{~d} y)\right]\right|_{\gamma=\gamma^{*}}
$$

To this end, it is enough to check that the partial derivative on the right-hand side can be moved inside the expectation and the integration signs. But this is a simple consequence of the dominated convergence theorem, since

$$
\lim _{\Delta \rightarrow 0} \int_{\Omega} \int_{\mathbb{R}} \frac{e^{\left(\gamma^{*}+\Delta\right) y}-e^{\gamma^{*} y}}{\Delta} \mathcal{Z}_{t}(\mathrm{~d} y) \mathrm{d} \mathbb{P}=\lim _{\Delta \rightarrow 0} \int_{\Omega} \int_{\mathbb{R}} \frac{e^{\Delta y}-1}{\Delta} e^{\gamma^{*} y} \mathcal{Z}_{t}(\mathrm{~d} y) \mathrm{d} \mathbb{P},
$$

and the absolute value of the integrand is bounded by the integrable function

$$
y \mapsto e^{\left(\gamma^{*}+\varepsilon\right) y} \mathbf{1}_{\{y \geqslant 0\}}+e^{\left(\gamma^{*}-\varepsilon\right) y} \mathbf{1}_{\{y<0\}}
$$

for sufficiently small $\Delta$ and all $y \in \mathbb{R}$.

Using formula (3.1) we derive

$$
\mathbb{E}\left[\sum_{k=1}^{\mathcal{Y}_{t}} e^{\gamma^{*} z_{k, t}} z_{k, t}\right]=\left.\frac{\partial}{\partial \gamma}\left(e^{t \Phi(\gamma)}\right)\right|_{\gamma=\gamma^{*}}=t \Phi^{\prime}\left(\gamma^{*}\right) e^{t \Phi\left(\gamma^{*}\right)}, \quad t \geqslant 0
$$

This immediately yields

$$
\mathbb{E}\left[\sum_{k=1}^{\mathcal{Y}_{t}} e^{\gamma^{*} z_{k, t}^{\circ}} z_{k, t}^{\circ}\right]=e^{-t \Phi\left(\gamma^{*}\right)}\left(t \Phi^{\prime}\left(\gamma^{*}\right) e^{t \Phi\left(\gamma^{*}\right)}\right)-t \mu\left(\gamma^{*}\right) \mathbb{E} \mathcal{M}_{t}\left(\gamma^{*}\right)=t\left(\Phi^{\prime}\left(\gamma^{*}\right)-\mu\left(\gamma^{*}\right)\right)=0 .
$$


The second claim in (3.7) follows from the formula

$$
\mathbb{E}\left[\sum_{k=1}^{\mathcal{Y}_{t}} e^{\gamma^{*} z_{k, t}} z_{k, t}^{2}\right]=\left.\frac{\partial^{2}}{\partial \gamma^{2}}\left[\mathbb{E} \int_{\mathbb{R}} e^{\gamma y} \mathcal{Z}_{t}(\mathrm{~d} y)\right]\right|_{\gamma=\gamma^{*}},
$$

which can be proved similarly. The proof is complete.

Remark 3.8. As has been pointed out by the referee Lemma 3.6 also follows from the many-to-one lemma for continuous-time branching random walks.

Lemma 3.9. Assume that $\gamma^{*}<s_{\infty}$. Then for every fixed $t \geqslant 0$ and $\delta>0$ such that $(1+\delta) \gamma^{*}<s_{\infty}$ we have

$$
\mathbb{E}\left[\left(\sum_{k=1}^{\mathcal{Y}_{t}} e^{\gamma^{*} z_{k, t}^{\circ}}\right)^{1+\delta}\right]<\infty \quad \text { and } \quad \mathbb{E}\left[\left(\sum_{k=1}^{\mathcal{Y}_{t}} e^{\gamma^{*} z_{k, t}^{\circ}}\left(z_{k, t}\right)_{+}\right)^{1+\delta}\right]<\infty,
$$

where $x_{+}:=\max (x, 0)$.

Proof. Let us prove the first claim. Using the inequality

$$
\left(\sum_{k=1}^{n} x_{k}\right)^{1+\delta} \leqslant n^{\delta}\left(\sum_{k=1}^{n} x_{k}^{1+\delta}\right)
$$

which holds for $n \in \mathbb{N}$ and arbitrary nonnegative reals $x_{1}, x_{2}, \ldots, x_{n}$, we infer

$$
\mathbb{E}\left(\sum_{k=1}^{\mathcal{Y}_{t}} e^{\gamma^{*} z_{k, t}}\right)^{1+\delta} \leqslant \mathbb{E}\left(\mathcal{Y}_{t}^{\delta} \sum_{k=1}^{\mathcal{Y}_{t}} e^{(1+\delta) \gamma^{*} z_{k, t}}\right)=\sum_{k=1}^{\infty} \mathbb{E}\left(\mathcal{Y}_{t}^{\delta} e^{(1+\delta) \gamma^{*} z_{k, t}} \mathbf{1}_{\left\{k \leqslant \mathcal{Y}_{t}\right\}}\right) .
$$

Pick $p>1$ such that $p(1+\delta) \gamma^{*}<s_{\infty}$ and $q>1$ such that $1 / p+1 / q=1$. By Hölder's inequality we obtain

$$
\mathbb{E}\left(\mathcal{Y}_{t}^{\delta} e^{(1+\delta) \gamma^{*} z_{k, t}} \mathbf{1}_{\left\{k \leqslant \mathcal{Y}_{t}\right\}}\right) \leqslant\left(\mathbb{E} e^{p(1+\delta) \gamma^{*} z_{k, t}} \mathbf{1}_{\left\{k \leqslant \mathcal{Y}_{t}\right\}}\right)^{1 / p}\left(\mathbb{E} \mathcal{Y}_{t}^{q \delta} \mathbf{1}_{\left\{k \leqslant \mathcal{Y}_{t}\right\}}\right)^{1 / q}
$$

and thereupon

$$
\begin{aligned}
& \mathbb{E}\left(\sum_{k=1}^{\mathcal{Y}_{t}} e^{\gamma^{*} z_{k, t}}\right)^{1+\delta} \leqslant \sum_{k=1}^{\infty}\left(\mathbb{E} e^{p(1+\delta) \gamma^{*} z_{k, t}} \mathbf{1}_{\left\{k \leqslant \mathcal{Y}_{t}\right\}}\right)^{1 / p}\left(\mathbb{E} \mathcal{Y}_{t}^{q \delta} \mathbf{1}_{\left\{k \leqslant \mathcal{Y}_{t}\right\}}\right)^{1 / q} \\
& \leqslant\left(\sum_{k=1}^{\infty} \mathbb{E} e^{p(1+\delta) \gamma^{*} z_{k, t}} \mathbf{1}_{\left\{k \leqslant \mathcal{Y}_{t}\right\}}\right)^{1 / p}\left(\sum_{k=1}^{\infty} \mathbb{E} \mathcal{Y}_{t}^{q \delta} \mathbf{1}_{\left\{k \leqslant \mathcal{Y}_{t}\right\}}\right)^{1 / q}
\end{aligned}
$$

where the last passage is a consequence of Hölder's inequality for series. The first factor on the right-hand side is finite because $p(1+\delta) \gamma^{*}<s_{\infty}$ and

$$
\begin{aligned}
\sum_{k=1}^{\infty} \mathbb{E} e^{p(1+\delta) \gamma^{*} z_{k, t}} \mathbf{1}_{\{k \leqslant \mathcal{Y}}=\mathbb{E}\left(\sum_{k=1}^{\mathcal{Y}_{t}} e^{p(1+\delta) \gamma^{*} z_{k, t}}\right) & =\mathbb{E} \int_{\mathbb{R}} e^{p(1+\delta) \gamma^{*} y} \mathcal{Z}_{t}(\mathrm{~d} y) \\
& =e^{\Phi\left(p(1+\delta) \gamma^{*}\right) t} \mathbb{E} \mathcal{M}_{t}\left(p(1+\delta) \gamma^{*}\right)=e^{\Phi\left(p(1+\delta) \gamma^{*}\right) t}<\infty
\end{aligned}
$$


Formulae (2.2) and (2.3) imply that $\mathcal{Y}_{t}$ has exponential moment of some positive order for every fixed $t$. Therefore,

$$
\sum_{k=1}^{\infty} \mathbb{E} \mathcal{Y}_{t}^{q \delta} \mathbf{1}_{\left\{k \leqslant \mathcal{Y}_{t}\right\}}=\mathbb{E} \mathcal{Y}_{t}^{q \delta+1}<\infty
$$

and the proof of the first claim is complete.

To prove the second inequality we use exactly the same arguments to get the upper bound

$$
\begin{aligned}
& \mathbb{E}\left(\sum_{k=1}^{\mathcal{Y}_{t}} e^{\gamma^{*} z_{k, t}}\left(z_{k, t}\right)_{+}\right.)^{1+\delta} \\
& \leqslant\left(\sum_{k=1}^{\infty} \mathbb{E} e^{p(1+\delta) \gamma^{*} z_{k, t}\left(z_{k, t}\right)_{+}^{p(1+\delta)}} \mathbf{1}_{\left\{k \leqslant \mathcal{Y}_{t}\right\}}\right)^{1 / p}\left(\sum_{k=1}^{\infty} \mathbb{E} \mathcal{Y}_{t}^{q \delta} \mathbf{1}_{\left\{k \leqslant \mathcal{Y}_{t}\right\}}\right)^{1 / q} .
\end{aligned}
$$

It remains to note that

$$
\sum_{k=1}^{\infty} \mathbb{E} e^{p(1+\delta) \gamma^{*} z_{k, t}}\left(z_{k, t}\right)_{+}^{p(1+\delta)} \mathbf{1}_{\left\{k \leqslant \mathcal{Y}_{t}\right\}}=\mathbb{E} \int_{\mathbb{R}} e^{p(1+\delta) \gamma^{*} y} y_{+}^{p(1+\delta)} \mathcal{Z}_{t}(\mathrm{~d} y)<\infty,
$$

since $p(1+\delta) \gamma^{*}<s_{\infty}$. The proof is complete.

Proof of Proposition 3.2. Proof of PART (I). Fix $\theta>0$. Define a point process

$$
\Xi:=\sum_{k=1}^{\mathcal{Y}_{\theta}} \delta_{-\gamma^{*}} z_{k, \theta}^{\circ},
$$

and consider a discrete-time branching random walk $\left(\mathcal{Z}_{n}(\theta)\right)_{n=0,1,2, \ldots}$, where

$$
\mathcal{Z}_{n}(\theta):=\sum_{k=1}^{\mathcal{Y}_{n \theta}} \delta_{-\gamma^{*} z_{k, n \theta}^{\circ}}, \quad n=0,1,2, \ldots
$$

The discrete-time branching random walk $\left(\mathcal{Z}_{k}(\theta)\right)$ has the displacement process $\Xi$ and satisfies the following three conditions:

$$
\begin{aligned}
\mathbb{E}\left(\int_{\mathbb{R}} e^{-y} \mathcal{Z}_{1}(\theta)(\mathrm{d} y)\right)=1, \quad \mathbb{E}\left(\int_{\mathbb{R}} e^{-y} y \mathcal{Z}_{1}(\theta)(\mathrm{d} y)\right)=0 \text { and } \\
\mathbb{E}\left(\int_{\mathbb{R}} e^{-y} y^{2} \mathcal{Z}_{1}(\theta)(\mathrm{d} y)\right)=\theta\left(\gamma^{*}\right)^{2} \Phi^{\prime \prime}\left(\gamma^{*}\right)<\infty,
\end{aligned}
$$

where the last two relations are secured by Lemma 3.6. Moreover, Lemma 3.9 yields

$$
\mathbb{E}\left(\int_{\mathbb{R}} e^{-y} \mathcal{Z}_{1}(\theta)(\mathrm{d} y)\right)^{1+\delta}<\infty \text { and } \mathbb{E}\left(\int_{\mathbb{R}} e^{-y} y_{+} \mathcal{Z}_{1}(\theta)(\mathrm{d} y)\right)^{1+\delta}<\infty,
$$

whence conditions (5.3) in [31] hold. Therefore, Assumption $(\mathrm{H})$ in the same reference holds for the discrete-time branching random walk $\left(\mathcal{Z}_{n}(\theta)\right)_{n=0,1,2, \ldots}$ for every fixed $\theta>0$. 
In particular, by Theorem 5.29 in [31, see also Theorem 1.1 in [2], we have

$$
\begin{aligned}
\sqrt{n} \mathcal{M}_{n}\left(\gamma^{*}\right)=\sqrt{n} \sum_{k=1}^{\mathcal{Y}_{n}} e^{\gamma^{*} z_{k, n}^{\circ}}=\sqrt{n} \int_{\mathbb{R}} e^{-y} \mathcal{Z}_{n}(1)(\mathrm{d} y) \\
\stackrel{\mathbb{P}}{\rightarrow} \sqrt{\frac{2}{\pi\left(\gamma^{*}\right)^{2} \Phi^{\prime \prime}\left(\gamma^{*}\right)}} D_{\infty}=: D, \quad n \rightarrow \infty,
\end{aligned}
$$

where $D$ is a.s. positive, because in our settings the process does not extinct with probability one. It remains to show the convergence in probability to $D$ along $t \rightarrow \infty, t \in \mathbb{R}$. This can be accomplished by adopting the argument given on p. 47 in [12] as follows. From (3.12) we know that for every fixed $x>0$

$$
\left(\sqrt{n+1} \mathcal{M}_{n+1}\left(\gamma^{*}\right)-\sqrt{n} \mathcal{M}_{n}\left(\gamma^{*}\right)\right) \mathbb{1}_{\left\{\sqrt{n} \mathcal{M}_{n}\left(\gamma^{*}\right) \leqslant x\right\}} \stackrel{\mathbb{P}}{\rightarrow} 0, \quad n \rightarrow \infty,
$$

and therefore by the dominated convergence theorem we have for every $u \geqslant 0$

$$
\mathbb{E} \exp \left(-u\left(\left(\sqrt{n+1} \mathcal{M}_{n+1}\left(\gamma^{*}\right)-\sqrt{n} \mathcal{M}_{n}\left(\gamma^{*}\right)\right) \mathbb{1}_{\left\{\sqrt{n} \mathcal{M}_{n}\left(\gamma^{*}\right) \leqslant x\right\}}\right)\right) \rightarrow 1, \quad n \rightarrow \infty .
$$

Further, by the martingale property of $\left(\mathcal{M}_{t}\left(\gamma^{*}\right)\right)$ and applying Jensen's inequality twice to the convex function $x \mapsto \exp (-u x)$ we obtain for every $t \geqslant 0$

$$
\begin{aligned}
& \mathbb{E} \exp \left(-u\left(\left(\sqrt{[t]+1} \mathcal{M}_{[t]+1}\left(\gamma^{*}\right)-\sqrt{[t]} \mathcal{M}_{[t]}\left(\gamma^{*}\right)\right) \mathbb{1}_{\left\{\sqrt{[t]} \mathcal{M}_{[t]}\left(\gamma^{*}\right) \leqslant x\right\}}\right)\right) \\
& =\mathbb{E}\left[\mathbb{E}\left\{\exp \left(-u\left(\left(\sqrt{[t]+1} \mathcal{M}_{[t]+1}\left(\gamma^{*}\right)-\sqrt{[t]} \mathcal{M}_{[t]}\left(\gamma^{*}\right)\right) \mathbb{1}_{\left\{\sqrt{[t]} \mathcal{M}_{[t]}\left(\gamma^{*}\right) \leqslant x\right\}}\right)\right) \mid \mathcal{F}_{t}\right\}\right] \\
& \geqslant \mathbb{E}\left\{\exp \left(-u\left(\left(\sqrt{[t]+1} \mathcal{M}_{t}\left(\gamma^{*}\right)-\sqrt{[t]} \mathcal{M}_{[t]}\left(\gamma^{*}\right)\right) \mathbb{1}_{\left\{\sqrt{[t]} \mathcal{M}_{[t]}\left(\gamma^{*}\right) \leqslant x\right\}}\right)\right)\right\} \\
& \left.=\mathbb{E}\left[\mathbb{E}\left\{\exp \left(-u\left(\left(\sqrt{[t]+1} \mathcal{M}_{t}\left(\gamma^{*}\right)-\sqrt{[t]} \mathcal{M}_{[t]}\left(\gamma^{*}\right)\right) \mathbb{1}_{\left\{\sqrt{[t]} \mathcal{M}_{[t]}\left(\gamma^{*}\right) \leqslant x\right\}}\right)\right) \mid \mathcal{F}_{[t]\}]}\right\}\right)\right\} . \\
& \geqslant \mathbb{E}\left\{\exp \left(-u\left(\left(\sqrt{[t]+1} \mathcal{M}_{[t]}\left(\gamma^{*}\right)-\sqrt{[t]} \mathcal{M}_{[t]}\left(\gamma^{*}\right)\right) \mathbb{1}_{\left\{\sqrt{[t]} \mathcal{M}_{[t]}\left(\gamma^{*}\right) \leqslant x\right\}}\right)\right) .\right.
\end{aligned}
$$

Sending $t \rightarrow \infty$ in the above inequalities we obtain

$$
\left(\sqrt{[t]+1} \mathcal{M}_{t}\left(\gamma^{*}\right)-\sqrt{[t]} \mathcal{M}_{[t]}\left(\gamma^{*}\right)\right) \mathbb{1}_{\left\{\sqrt{[t]} \mathcal{M}_{[t]}\left(\gamma^{*}\right) \leqslant x\right\}} \stackrel{\mathbb{P}}{\rightarrow} 0, \quad t \rightarrow \infty .
$$

By the triangle inequality

$$
\begin{aligned}
\left|\sqrt{t} \mathcal{M}_{t}\left(\gamma^{*}\right)-D\right| & \leqslant\left|\sqrt{t} \mathcal{M}_{t}\left(\gamma^{*}\right)-\sqrt{[t]+1} \mathcal{M}_{t}\left(\gamma^{*}\right)\right| \\
& +\left|\sqrt{[t]+1} \mathcal{M}_{t}\left(\gamma^{*}\right)-\sqrt{[t]} \mathcal{M}_{[t]}\left(\gamma^{*}\right)\right| \mathbb{1}_{\left\{\sqrt{[t]} \mathcal{M}_{[t]}\left(\gamma^{*}\right) \leqslant x\right\}} \\
& +\left|\sqrt{[t]+1} \mathcal{M}_{t}\left(\gamma^{*}\right)-\sqrt{[t]} \mathcal{M}_{[t]}\left(\gamma^{*}\right)\right| \mathbb{1}_{\left\{\sqrt{[t]} \mathcal{M}_{[t]}\left(\gamma^{*}\right)>x\right\}} \\
& +\left|\sqrt{[t]} \mathcal{M}_{[t]}\left(\gamma^{*}\right)-D\right| .
\end{aligned}
$$

The second and fourth summands converge to zero in probability as $t \rightarrow \infty$ by (3.13) and (3.12), respectively. The first summand does this by Markov's inequality since $\sqrt{t+1}-$ $\sqrt{t} \rightarrow 0$ as $t \rightarrow \infty$. The probability that the third summand is larger than some $\delta>0$ is bounded from above by $\mathbb{P}\left\{\sqrt{[t]} \mathcal{M}_{[t]}\left(\gamma^{*}\right)>x\right\}$ which can be made arbitrarily small by 
choosing $x$ large enough in view of (3.12) and a.s. finiteness of $D$. This completes the proof of convergence in part (i).

Let us show that $D_{\infty}$ (and also $D$ ) satisfies (1.5). Let $\tau_{1}$ be the time of the first split in $\left(\mathcal{Z}_{t}\right)_{t \geqslant 0}$, then

$$
\mathcal{Z}_{t}(\cdot) \stackrel{d}{=} \mathbf{1}_{\left\{\tau_{1}>t\right\}} \delta_{0}(\cdot)+\mathbf{1}_{\left\{\tau_{1} \leqslant t\right\}} \sum_{k=1}^{N} \mathcal{Z}_{t-\tau_{1}}^{(k)}\left(\cdot-z_{k, \tau_{1}}\right),
$$

and therefore

$$
\begin{aligned}
\sqrt{t} \mathcal{M}_{t}\left(\gamma^{*}\right) & =\sqrt{t} e^{-\Phi\left(\gamma^{*}\right) t} \int_{\mathbb{R}} e^{\gamma^{*} y} \mathcal{Z}_{t}(\mathrm{~d} y) \stackrel{d}{=} \mathbf{1}_{\left\{\tau_{1}>t\right\}} \sqrt{t} e^{-\Phi\left(\gamma^{*}\right) t} \\
& +\mathbf{1}_{\left\{\tau_{1} \leqslant t\right\}} e^{-\Phi\left(\gamma^{*}\right) \tau_{1}} \sum_{k=1}^{N} \sqrt{t} e^{-\Phi\left(\gamma^{*}\right)\left(t-\tau_{1}\right)} A_{k}^{\gamma^{*}} \int_{\mathbb{R}} e^{\gamma^{*} y} \mathcal{Z}_{t-\tau_{1}}^{(k)}(\mathrm{d} y) .
\end{aligned}
$$

Sending $t \rightarrow \infty$ yields (1.5) because $\tau_{1}$ has the standard exponential law and is independent of $\left(\mathcal{Z}_{t}^{(k)}\right)_{t \geqslant 0}, k \in \mathbb{N}$. The proof of part (i) is complete.

PROOF OF PART (II). The claim of part (ii) can be reformulated as follows:

$$
\min _{k=1, \ldots, \mathcal{Y}_{t}}\left(-\gamma^{*} z_{k, t}^{\circ}\right)-\frac{1}{2} \log t \stackrel{\mathbb{P}}{\rightarrow}+\infty, \quad t \rightarrow \infty
$$

Fix arbitrary $M>0$ and define a function

$$
p_{M}(t):=\mathbb{P}\left\{\min _{k=1, \ldots, \mathcal{Y}_{t}}\left(-\gamma^{*} z_{k, t}^{\circ}\right)-\frac{1}{2} \log t<M\right\}, \quad t \geqslant 0 .
$$

By Theorem 5.12 in [31] applied to the discrete-time branching random walk $\left(\mathcal{Z}_{n}^{(\theta)}\right)_{n=0,1,2, \ldots \text {, }}$ see also [1, 22, we already know that

$$
\lim _{n \rightarrow \infty} p_{M}(n \theta)=0
$$

for every fixed $\theta>0$. In order to finish the proof of part (ii) it remains to show that

$$
\lim _{t \rightarrow \infty, t \in \mathbb{R}} p_{M}(t)=0
$$

According to the Croft-Kingman lemma, see Corollary 2 in [27], it is enough to check that $t \mapsto p_{M}(t)$ is right-continuous. To prove the latter statement, note that for $0 \leqslant s \leqslant t$ we have

$$
\begin{aligned}
& \left|p_{M}(t)-p_{M}(s)\right| \leqslant \mathbb{P}\{\text { there are splits during }[s, t]\} \\
& \qquad+\mathbb{P}\left\{\min _{k=1, \ldots, \mathcal{Y}_{t}}\left(-\gamma^{*} z_{k, t}^{\circ}\right) \in\left[M+\frac{1}{2} \log s, M+\frac{1}{2} \log t\right)\right\}
\end{aligned}
$$

where we have used the equality $\min _{k=1, \ldots, \mathcal{Y}_{t}}\left(-\gamma^{*} z_{k, t}^{\circ}\right)=\min _{k=1, \ldots, \mathcal{Y}_{s}}\left(-\gamma^{*} z_{k, s}^{\circ}\right)$ which holds if there are no splits in $[s, t]$. The right-hand side of the last display converges to 0 as $s \rightarrow t+$. The proof of part (ii) is complete. 


\section{Proof of Theorem 1.4}

The key ingredient in the proof is Propostion 3.2 and the following lemma.

Lemma 4.1. Assume that $\left(r_{t}\right)_{t \geqslant 0}$ is an integer-valued random process such that $r_{t} \stackrel{\mathbb{P}}{\rightarrow} \infty$, as $t \rightarrow \infty$. Further, suppose that for every $t \geqslant 0$ there is an array $\left(a_{k, t}\right)_{k=1, \ldots, r_{t}}$ of a.s. positive random weights such that

$$
\sum_{k=1}^{r_{t}} a_{k, t}^{\gamma} \stackrel{\mathbb{P}}{\rightarrow} a_{\infty} \quad \text { and } \quad \max _{k=1, \ldots, r_{t}} a_{k, t} \stackrel{\mathbb{P}}{\rightarrow} 0, \quad t \rightarrow \infty
$$

for some a.s. positive random variable $a_{\infty}$ and $\gamma \in(0,2]$. Let $\left(X_{k}\right)_{k \in \mathbb{N}}$ be a sequence of independent random variables with common distribution function $F_{0}$ satisfying $\left(H_{\gamma}\right)$ and which are independent of $\left(a_{k, t}\right)_{k=1, \ldots, r_{t}}$ and $r_{t}$ for every fixed $t \geqslant 0$. Put

$$
S_{t}:=\sum_{k=1}^{r_{t}} a_{k, t} X_{k}, \quad t \geqslant 0 .
$$

Then

$$
\lim _{t \rightarrow \infty} \mathbb{E} \exp \left(i \xi S_{t}\right)=\mathbb{E} \widehat{g}_{\gamma}\left(\xi a_{\infty}^{1 / \gamma}\right), \quad \xi \in \mathbb{R}
$$

where $\widehat{g}_{\gamma}$ is defined by (1.3).

Proof. The proof is based on the following asymptotic expansions of the characteristic function $\phi_{0}$ of $X_{0}$, that are equivalent to the corresponding assumptions of the distribution function $F_{0}$, see Theorem 2.6.5 in [23]:

- if the case (a) of $\left(H_{1}\right)$ holds, then $\log \phi_{0}(\xi)=i m_{0} \xi+o(\xi)$ as $\xi \rightarrow 0$;

- if the case (b) of $\left(H_{1}\right)$ holds, then $\log \phi_{0}(\xi)=i m_{0} \xi-\pi c_{0}^{+}|\xi|+o(\xi)$ as $\xi \rightarrow 0$;

- if $\left(H_{2}\right)$ holds, then $\log \phi_{0}(\xi)=-\frac{\sigma_{0}^{2}}{2} \xi^{2}+o\left(\xi^{2}\right)$ as $\xi \rightarrow 0$;

- if $\left(H_{\gamma}\right)$ holds with $\gamma \in(0,1) \cup(1,2)$, then

$$
\log \phi_{0}(\xi)=-k_{0}|\xi|^{\gamma}\left(1-i \eta_{0} \tan (\pi \gamma / 2) \operatorname{sign} \xi\right)+o\left(|\xi|^{\gamma}\right), \quad \xi \rightarrow 0 .
$$

Using the above expansions the rest of the proof is standard and relies on the formula

$$
\mathbb{E} \exp \left(i \xi S_{t}\right)=\mathbb{E}\left(\exp \left(\sum_{k=1}^{r_{t}} \log \phi_{0}\left(a_{k, t} \xi\right)\right)\right), \quad \xi \in \mathbb{R}, \quad t \geqslant 0 .
$$

We will give full details in the case (b) of $\left(H_{1}\right)$. The other cases can be checked similarly. From the equality

$$
\begin{aligned}
\mathbb{E} \exp \left(i \xi S_{t}\right)=\mathbb{E}\left(\operatorname { e x p } \left(\sum_{k=1}^{r_{t}}\left(\log \phi_{0}\left(a_{k, t} \xi\right)-i m_{0} \xi a_{k, t}+\pi c_{0}^{+}|\xi| a_{k, t}\right)\right.\right. & \\
& \left.\left.+i m_{0} \xi \sum_{k=1}^{r_{t}} a_{k, t}-\pi c_{0}^{+}|\xi| \sum_{k=1}^{r_{t}} a_{k, t}\right)\right), \quad \xi \in \mathbb{R}, \quad t \geqslant 0
\end{aligned}
$$

we see that it is enough to check that for every fixed $\xi \in \mathbb{R}$

$$
\sum_{k=1}^{r_{t}}\left(\log \phi_{0}\left(a_{k, t} \xi\right)-i m_{0} \xi a_{k, t}+\pi c_{0}^{+}|\xi| \sum_{k=1}^{r_{t}} a_{k, t}\right) \stackrel{\mathbb{P}}{\rightarrow} 0, \quad t \rightarrow \infty .
$$


Fix $\varepsilon>0$. There exists $x_{0}(\varepsilon)>0$ such that

$$
\left|\log \phi_{0}(x)-i m_{0} x+\pi c_{0}^{+}\right| x|| \leqslant \varepsilon|x|, \quad|x| \leqslant x_{0}(\varepsilon) .
$$

Therefore, for every fixed $\varepsilon_{0}>0$

$$
\begin{aligned}
& \mathbb{P}\left\{\sum_{k=1}^{r_{t}}\left|\log \phi_{0}\left(a_{k, t} \xi\right)-i m_{0} \xi a_{k, t}+\pi c_{0}^{+}\right| \xi\left|a_{k, t}\right|>\varepsilon_{0}\right\} \\
& \quad \leqslant \mathbb{P}\left\{\varepsilon|\xi| \sum_{k=1}^{r_{t}} a_{k, t}>\varepsilon_{0}\right\}+\mathbb{P}\left\{|\xi| a_{k, t}>x_{0}(\varepsilon) \text { for some } k=1, \ldots, r_{t}\right\} \\
& \quad=\mathbb{P}\left\{\varepsilon|\xi| \sum_{k=1}^{r_{t}} a_{k, t}>\varepsilon_{0}\right\}+\mathbb{P}\left\{|\xi| \max _{k=1, \ldots, r_{t}} a_{k, t}>x_{0}(\varepsilon)\right\} .
\end{aligned}
$$

Sending $t \rightarrow \infty$ and then $\varepsilon \rightarrow+0$ yields (4.2). The proof is complete.

Proof of Theorem 1.4. Put $a_{k, t}:=t^{\frac{1}{2 \gamma^{*}}} e^{z_{k, t}-t \frac{\Phi\left(\gamma^{*}\right)}{\gamma^{*}}}, r_{t}:=\mathcal{Y}_{t}, a_{\infty}:=D^{\prime}=\sqrt{\frac{2}{\pi\left(\gamma^{*}\right)^{2} \Phi^{\prime \prime}\left(\gamma^{*}\right)}} D_{\infty}$, $\gamma=\gamma^{*}$, and finally

$$
S_{t}=t^{\frac{1}{2 \gamma^{*}}} e^{-\mu\left(\gamma^{*}\right) t} \sum_{k=1}^{\mathcal{Y}_{t}} e^{z_{k, t}} X_{k}, \quad t \geqslant 0
$$

From Proposition 3.2 we know that all the assumptions of Lemma 4.1 hold and therefore

$$
\lim _{t \rightarrow \infty} \mathbb{E} \exp \left(i \xi S_{t}\right)=\mathbb{E} \widehat{g}_{\gamma}\left(\xi\left(\sqrt{\frac{2}{\pi\left(\gamma^{*}\right)^{2} \Phi^{\prime \prime}\left(\gamma^{*}\right)}} D_{\infty}\right)^{1 / \gamma}\right), \quad \xi \in \mathbb{R}
$$

By Proposition 2.5

$$
\mathbb{E} \exp \left(i \xi S_{t}\right)=\phi\left(t, t^{\frac{1}{2 \gamma^{*}}} e^{-\mu\left(\gamma^{*}\right) t} \xi\right)
$$

which proves convergence. The fact that $D_{\infty}$ satisfies (1.5) has already been proved above. The proof of Theorem 1.4 is complete.

\section{ACKNOWLEDGMENT}

We thank two anonymous referees for the detailed and useful reports containing numerous remarks and suggestions.

\section{REFERENCES}

[1] AїDÉKon, E. AND SHI, Z. (2010). Weak convergence for the minimal position in a branching random walk: a simple proof. Periodica Mathematica Hungarica, 61(1-2), 43-54.

[2] AïdÉkon, E. AND Shi, Z. (2014). The Seneta-Heyde scaling for the branching random walk. Ann. Probab., 42(3), 959-993.

[3] Athreya, K. B. And Ney, P. E. (1972). Branching Processes. Springer-Verlag Berlin, Heidelberg, New York.

[4] Bassetti, F. And Ladelli, L. (2012). Self-similar solutions in one-dimensional kinetic models: A probabilistic view. Ann. Appl. Probab., 22(5), 1928-1961.

[5] Bassetti, F., Ladelli, L. And Matthes, D. (2011). Central limit theorem for a class of onedimensional kinetic equations. Probab. Theory Related Fields, 150(1-2), 77-109. 
[6] Bassetti, F., Ladelli, L. And Matthes, D. (2015). Infinite energy solutions to inelastic homogeneous Boltzmann equations. Electron. J. Probab., 20, paper no. 89, 1-34.

[7] Bassetti, F. And Perversi, E. (2013). Speed of convergence to equilibrium in Wasserstein metrics for Kac-like kinetic equations. Electron. J. Probab., 18, paper no. 6, 1-35.

[8] Barral, J., Rhodes, R. And Vargas, V. (2012). Limiting laws of supercritical branching random walks. C. R. Acad. Sci. Paris, Ser. I, 350, 535-538.

[9] Bertoin, J. And Mallein, B.(2018). Biggins martingale convergence for branching Léevy processes. Electron. J. Probab., 23, paper no. 83, 1-12.

[10] Biggins, J. (1977). Martingale convergence in the branching random walk. J. Appl. Probab., 14(1), 25-37.

[11] Biggins, J. (1992). Uniform convergence of martingales in the branching random walk. Ann. Probab., 20(1), 137-151.

[12] Biggins, J. And Kyprianou, A. (1996). Branching Random Walk: Seneta-Heyde norming. In Trees: Proceedings of a Workshop, Versailles June 14-16, 1995 (B. Chauvin, S. Cohen and A. Rouault, eds.). Birkhäuser, Basel.

[13] Biggins, J. And Kyprianou, A. (2005). Fixed points of the smoothing transform: the boundary case. Electron. J. Probab., 10, paper no. 17, 609-631.

[14] Bobylev, A. V. And Cercignani, C. (2003). Self-similar asymptotics for the Boltzmann equation with inelastic and elastic interactions. J. Stat. Phys., 110, 333-375.

[15] Bobylev, A. V., Cercignani, C. And Gamba, I. M. (2009). On the self-similar asymptotics for generalized nonlinear kinetic Maxwell models. Comm. Math. Phys., 291, 599-644.

[16] Carlen, E. A., Carvalho, M. C. and Gabetta, E. (2000). Central limit theorem for Maxwellian molecules and truncation of the Wild expansion. Comm. Pure Appl. Math., 53, 370-397.

[17] Chauvin, B., Klein, T., Marckert, J-F. And Rounult, A. (2004). Martingales and Profile of Binary Search Trees. Electron. J. Probab., 10, paper no. 12, 1-27.

[18] Dadoun, B. (2017). Asymptotic of self-similar growth-fragmentation processes. Electron. J. Probab., 22, paper no. 27, 1-30.

[19] Feller, W. (1971). An introduction to probability theory and its applications. Vol. II. Second edition John Wiley \& Sons, Inc., New York-London-Sydney.

[20] Gabetta, E., Regazzini, E. (2006). Some new results for McKean's graphs with applications to Kac's equation. J. Stat. Phys., 125, 943-970.

[21] Gabetta, E., Regazzini, E. (2008). Central limit theorem for solutions of the Kac equation. Ann. Appl. Probab., 18(6), 2320-2336.

[22] Hu, Y., SHI, Z.(2009). Minimal position and critical martingale convergence in branching random walks, and directed polymers on disordered trees. Ann. Probab., 37(2), 742-789.

[23] Ibragimov, I. And Linnik, Yu. (1971). Independent and Stationary Sequences of random variables. Wolters-Noordhoff Publishing, Groningen.

[24] Kabluchko, Z., Marynych, A. And Sulzbach, H. (2017). General Edgeworth expansions with applications to profiles of random trees. Ann. Appl. Probab., 27(6), 3478-3524.

[25] KAC, M. (1956). Foundations of kinetic theory. In Proceedings of the Third Berkeley Symposium on Mathematical Statistics and Probability, 1954-1955, Vol. III 171-197. Univ. California Press, Berkeley, CA.

[26] KIELEK, Z. (1988). An application of the convolution iterates to evolution equation in Banach space. Univ. Iagel. Acta Math., 27, 247-257.

[27] Kingman, J. F. C.(1963). Ergodic properties of continuous-time Markov processes and their discrete skeletons. Proc. London Math. Soc., 13, 593-604.

[28] Matthes, D. And Toschni, G. (2008). On steady distributions of kinetic models of conservative economies. J. Stat. Phys., 130, 1087-1117.

[29] McKean, H. P. (1966). Speed of approach to equilibrium for Kacs caricature of a Maxwellian gas. Arch. Ration. Mech. Anal., 21, 343-367.

[30] Pareschi, L. And Toscani, G. (2006). Self-similarity and power-like tails in nonconservative kinetic models. J. Stat. Phys., 124, 747-779. 
[31] SHI, Z.(2015). Branching random walks. Lecture notes from the 42nd Probability Summer School held in Saint Flour, Springer.

[32] Uсhiyama, K. (1982). Spatial growth of a branching process of particles living in $\mathbf{R}^{d}$. Ann. Probab., 10, 896-918.

[33] Wild, E. (1951). On Boltzmanns equation in the kinetic theory of gases. Proc. Cambridge Philos. Soc., 47, 602-609.

Kamil Bogus, Faculty of Pure and Applied Mathematics, Wroceaw University of Science and Technology, ul. Wybrzeże Wyspiańskiego 27, 50-370 Wroceaw, Poland

E-mail address: kamil.bogus@pwr.edu.pl

Dariusz Buraczewski, Mathematical Institute, University of Wroceaw, Plac Grunwaldzki 2/4, 50-384 WrocŁaw, Poland

E-mail address: dbura@math.uni.wroc.pl

Alexander Marynych, Faculty of Computer Science and Cybernetics, Taras Shevchenko National University of Kyiv, 01601 Kyiv, Ukraine

E-mail address: marynych@unicyb.kiev.ua 